\title{
The Pion Electromagnetic Form Factor in the Meson Cloud Model
}

\author{
F. Carvalho ${ }^{1}$, F.O. Durães ${ }^{1,2}$, F.S. Navarra ${ }^{1}$, and M. Nielsen ${ }^{1}$ \\ ${ }^{1}$ Instituto de Física, Universidade de São Paulo, C.P. 66318, 05389-970 São Paulo, SP, Brazil \\ ${ }^{2}$ Dep. de Física, Faculdade de Ciências Biológicas, Exatas e Experimentais, \\ Universidade Presbiteriana Mackenzie, C.P. 01302-907 São Paulo, SP, Brazil
}

Received on 15 August, 2003.

\begin{abstract}
In this work we calculate the meson cloud effects on the pion electromagnetic form factor and perform a comparison with experimental data. We show that, even though the cloud is not the only non-perturbative process to be considered, its contribution is significant. We also study the influence of the cutoff choices on the results.
\end{abstract}

\section{Introduction}

The region between the perturbative and non-perturbative sector of QCD is the key for understanding the strong interaction. In this context, the pion electromagnetic form factor $\left(F_{\pi}\right)$ is of great interest. It is extremely well reproduced in the low momentum transfer $\left(Q^{2}\right)$ regime by the vector dominance model, and at high $Q^{2}$ by perturbative $\mathrm{QCD}$, which proposes the following asymptotic behavior for the $F_{\pi}$ function:

$$
Q^{2} F_{\pi}\left(q^{2}\right)=16 \pi f_{\pi}^{2} \alpha_{s}\left(Q^{2}\right) \quad\left(Q^{2} \rightarrow \infty\right)
$$

where $f_{\pi}$ is the pion decay constant.

From the comparison between the experimental data and the asymptotic behavior it is possible to determine the scale of momentum where the perturbative regime of QCD is reached. Recently, the pion electromagnetic form factor has been measured at momentum transfers $0.6 \mathrm{GeV}^{2} \leq Q^{2} \leq$ $1.6 \mathrm{GeV}^{2}$ by the Jefferson Laboratory $F_{\pi}$ collaboration. We can see that even at the highest experimentally accessible momenta, they are still very far from the asymptotic limit.

The main question here is: what are the non-perturbative effects responsible for the deviation from perturbative QCD at intermediate momenta? In this work we consider the contribution of the meson cloud around the pion to its eletromagnetic form factor.

The role of mesons in deep inelastic scattering (DIS) was first investigated by Sullivan [1]. He suggested that some fraction of the sea anti-quark distribution of the nucleon may be associated with the pion cloud around it. Several works developed this idea and gave origin to the meson cloud model (MCM). They are all based on the notion that the physical proton (p) may be expanded in a sum of virtual meson-baryon states (MB). This model has presented very meaningful results when applied to hadronic interactions $[2,3,4]$.

The success of the MCM in understanding particle production and asymmetries in open charm production leads us to expect it to play also an important role in hadron form factors.
Indeed, in a recent paper, G. Miller [5] presented a calculation of the electromagnetic form factor of the nucleon where the inclusion of meson cloud effects was needed to fit the experimental data, as shown in Fig. 1.

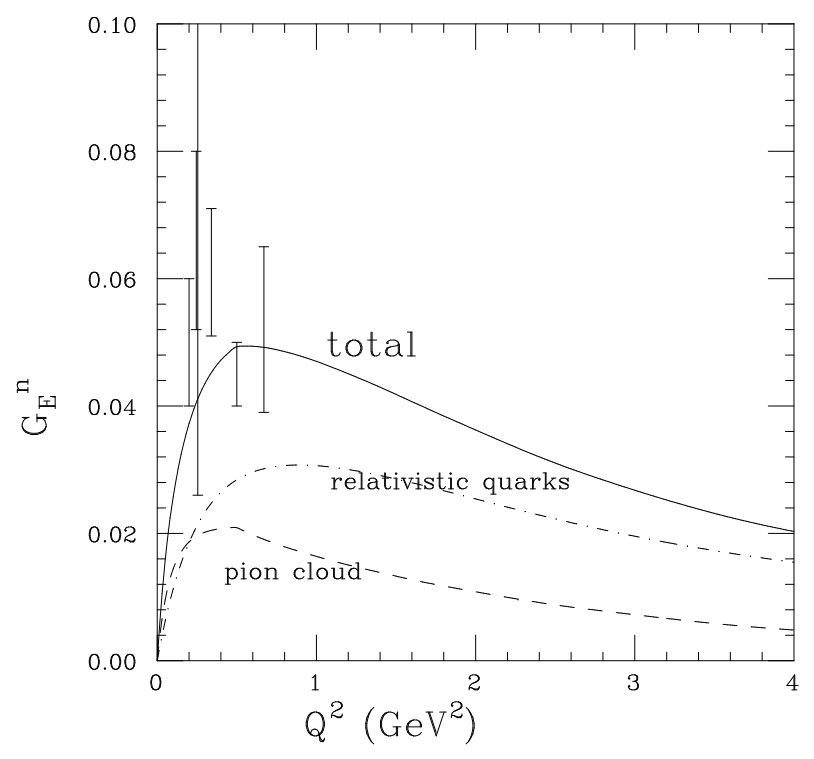

Figure 1. Nucleon electromagnetic form factor.

The purpose of this work is to perform an extension of the MCM to the pion and investigate the importance of the meson cloud in its electromagnetic form factor. The idea of describing a pion with cloud states has already been used by us previously in [6], where we have studied $D^{-} / D^{+}$asymmetry produced in $\pi p \rightarrow D X$ collisions. In that work we could see that the inclusion of the meson cloud leads to a good agreement with experimental data.

As in the case of the nucleon, we can represent the pion as a "bare pion" $\mid \pi^{-}>_{0}$ which can fluctuate in virtual Fock states composed by a pseudo-scalar $(\mathrm{P})$ meson and a vector meson (V), such as: 


$$
\begin{aligned}
\mid \pi^{-}> & =\left|\pi^{-}>_{0}+\right| \pi^{-} \rho^{0}>+\mid \pi^{0} \rho^{-}> \\
& +\left|K^{-} K^{0 *}>+\right| K^{0} K^{-*}> \\
& +\left|D^{-} D^{0 *}>+\right| D^{0} D^{-*}>+\ldots
\end{aligned}
$$

Thus, we can understand the photon-pion interaction as being an interaction between the photon and the constituents of the cloud. In Fig. 2, we show the interaction of the photon and the state $\mid P V>$
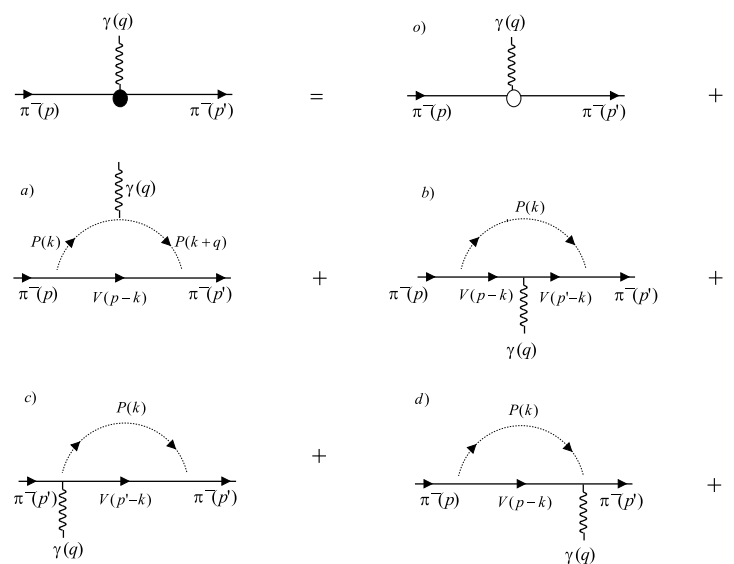

Figure 2. Photon-pion interaction in the MCM.

\section{The pion form factor}

In order to obtain the pion form factor we need to calculate the vertex function $\Gamma_{\mu}\left(p, p^{\prime}\right)$ for all the diagrams of Fig. 2 and for all the states of the equation (2).

In the case of the diagram $(a), \Gamma_{\mu}$ is given by

$$
\begin{array}{r}
i \Gamma_{\mu}\left(p, p^{\prime}\right)=i g^{2} Q_{\pi} \int \frac{d^{4} k}{(2 \pi)^{4}} F\left(k^{2}\right) F(k+q)^{2} \\
\frac{i}{k^{2}-m_{\pi}^{2}} \frac{i}{(k+q)^{2}-m_{\pi}^{2}}(2 k+q)_{\mu} \\
(-i) p_{\beta}^{\prime}(-i)\left[\frac{g^{\alpha \beta}-(p-k)^{\alpha}(p-k)^{\beta} / m_{\rho}^{2}}{(p-k)^{2}-m_{\rho}^{2}}\right] i p_{\alpha}
\end{array}
$$

For the internal vertex form factor (pion-meson-meson), we used the monopole parametrization:

$$
F\left(k^{2}\right)=\frac{m_{\pi}^{2}-\Lambda^{2}}{k^{2}-\Lambda^{2}}
$$

Using the expression

$$
\frac{1}{x^{2}-a^{2}}=-\int_{0}^{\infty} d \alpha e^{\alpha\left(x^{2}-a^{2}\right)}
$$

in each one of the five denominators, we arrive at

$$
\begin{aligned}
& i \Gamma_{\mu}\left(p, p^{\prime}\right)=g^{2} Q_{\rho}\left(m_{\pi}^{2}-\Lambda^{2}\right)^{2} \int \frac{d^{4} k}{(2 \pi)^{4}} \\
& \int_{0}^{\infty} \prod_{i=1}^{5} d \alpha_{i} e^{N} \exp \left(A k^{2}+2 B \cdot k\right) \\
& {\left[p \cdot p^{\prime}-\frac{1}{m_{\rho}^{2}}\left(m_{\pi}^{2} p \cdot p^{\prime}-k \cdot p p \cdot p^{\prime}-m_{\pi}^{2} k \cdot p^{\prime}+k \cdot p k \cdot p^{\prime}\right)\right.} \\
& \left.\left(2 k+p^{\prime}-p\right)_{\mu}\right]
\end{aligned}
$$

where

$$
\begin{aligned}
A= & \sum_{i=1}^{5} \alpha_{i}, \\
B= & -\left(\alpha_{2}+\alpha_{5}\right) p^{\prime}-\left(\alpha_{2}+\alpha_{3}+\alpha_{5}\right) p \quad \text { and } \\
N= & -\left(\alpha_{1}+\alpha_{2}\right) m_{\pi}^{2}+\alpha_{3}\left(m^{2}-\pi-m_{\rho}^{2}\right) \\
& \quad-\left(\alpha_{4}+\alpha_{5}\right) \Lambda^{2}+\left(\alpha_{2}+\alpha_{5}\right) q^{2}
\end{aligned}
$$

The advantage of arranging the terms in this way is that the $d^{4} k$ integration can be performed analytically. Doing this, we can group the terms proportional to $p_{\mu}$ and to $p_{\mu}^{\prime}$. Now these terms depend only on $Q^{2}=-q^{2}$ and on the $\alpha_{i}$ variables.

The integrals in $\alpha_{i}$ can be solved numerically. Performing them, the result is a function of $Q^{2}$, as we actually wish. So we have as a final result, some expressions like:

$$
\Gamma_{\mu}\left(p, p^{\prime}\right)=a\left(Q^{2}\right) p_{\mu}+b\left(Q^{2}\right) p_{\mu}^{\prime}
$$

Doing the same type of calculation for all the diagrams in Fig. 2, including the contributions of the other diagrams in equation (2) and adding their results we find:

$$
\Gamma_{\mu}\left(p, p^{\prime}\right)=F_{1}\left(Q^{2}\right) p_{\mu}+F_{2}\left(Q^{2}\right) p_{\mu}^{\prime}
$$

The pion electromagnetic form factor is associated with the term:

$$
F_{\pi}^{+}\left(Q^{2}\right)=\frac{F_{1}\left(Q^{2}\right)+F_{2}\left(Q^{2}\right)}{2}
$$

\section{Inputs for the calculations}

In order to evaluate the vertex functions derived in the previous section, it is necessary to know the value of the relevant masses, coupling constants $(g)$ and cutoff parameters $(\Lambda)$.

The $g_{\pi \pi \rho}, g_{\pi K K^{*}}$ and $g_{\pi D D^{*}}$ coupling constants have been measured experimentally [7, 8]. As the masses are all known, only the cutoff value remains as a free parameter. So far there is no systematic study which indicates the most appropriate cutoff value for the vertices involving pion-meson-meson fluctuations. Since we are using a 
monopole parametrization for these vertices, we have chosen cutoff values close to the corresponding vector meson masses.

In Table I we present all the values used in these calculations.

\begin{tabular}{|c|c|}
\hline$g_{\pi \pi \rho}$ & 12.16 \\
\hline$g_{\pi^{-} \pi^{-} \rho^{0}}=g_{\pi^{-} \pi^{0} \rho^{-}}$ & $\frac{1}{\sqrt{2}} g_{\pi \pi \rho}$ \\
\hline$g_{\pi K K^{*}}$ & 9.08 \\
\hline$g_{\pi^{-} K^{-} K^{0^{*}}}=g_{\pi^{-} K^{0} K^{-*}}$ & $g_{\pi K K^{*}}$ \\
\hline$g_{\pi D D^{*}}$ & 17.9 \\
\hline$g_{\pi^{-} D^{-} D^{0^{*}}}=g_{\pi^{-} D^{0} D^{-*}}$ & $g_{\pi D D^{*}}$ \\
\hline$\Lambda_{\pi \pi \rho}$ & $0.7-0.8 \mathrm{GeV}$ \\
\hline$\Lambda_{\pi K K^{*}}$ & $0.7-0.8 \mathrm{GeV}$ \\
\hline$\Lambda_{\pi D D^{*}}$ & $2.1 \mathrm{GeV}$ \\
\hline
\end{tabular}

Table I: Values of coupling constants and cutoff used in the vertex functions.

\section{Results and conclusions}

In Figs. 3 and 4 we present our results for $F_{\pi}\left(Q^{2}\right)$ with smaller and larger cutoff values respectively. In both figures we include experimental data from the $F_{\pi}$ collaboration [9], Amendolia et al. [10], from DESY [11] and from Bebeck et al. [12].

As it can be seen, the contribution from the meson cloud for the pion electromagnetic form factor is very important to understand the experimental data, specially in the intermediate momentum region. It is also possible to notice that there is a strong dependence of the results with the cutoff parameter.

As expected, we observe that the contribution from the $\mid \pi \rho>$ state is much larger than the others, due to the small masses involved. Surprisingly the $\mid D D^{*}>$ state gives a large contribution, which becomes more important at large $Q^{2}$. This is essentially due to the large value of the $D D^{*} \pi$ coupling. A possible interpretation of this fact is that at higher momenta, the photon can probe havier states.

Finally, we can compare the strength of the meson cloud in the pion (Figs. 3 and 4) and in the nucleon (Fig.1). We conclude that they have comparable magnitude, but in the case of the pion, the cloud is more important at intermediate momenta, while for the nucleon, the cloud becomes dominant at low momenta.

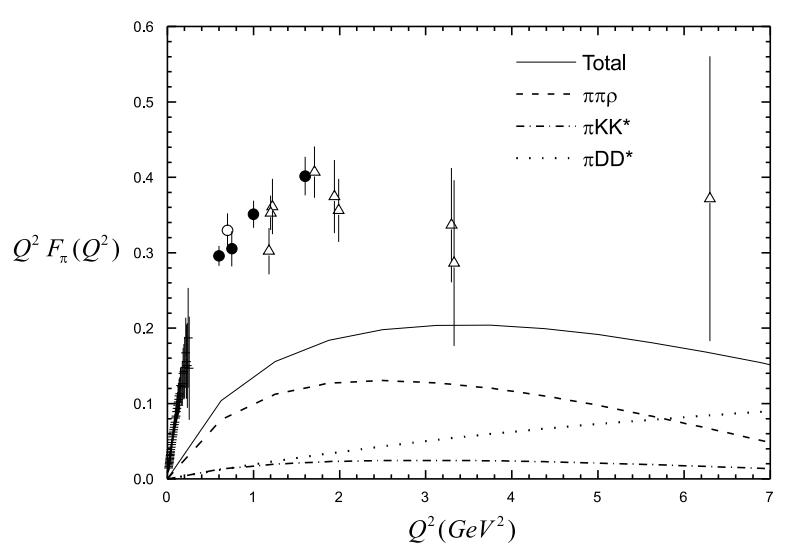

Figure 3. Comparison between the MCM and experimental data. The solid circles, cross, open circles and open triangles are the experimental data from refs. [9], [10], [11] and [12] respectively. In this figure we chose $\Lambda_{\pi \pi \rho}=\Lambda_{\pi K K^{*}}=0.7 \mathrm{GeV}$ and $\Lambda_{\pi D D^{*}}=2.1 \mathrm{GeV}$.

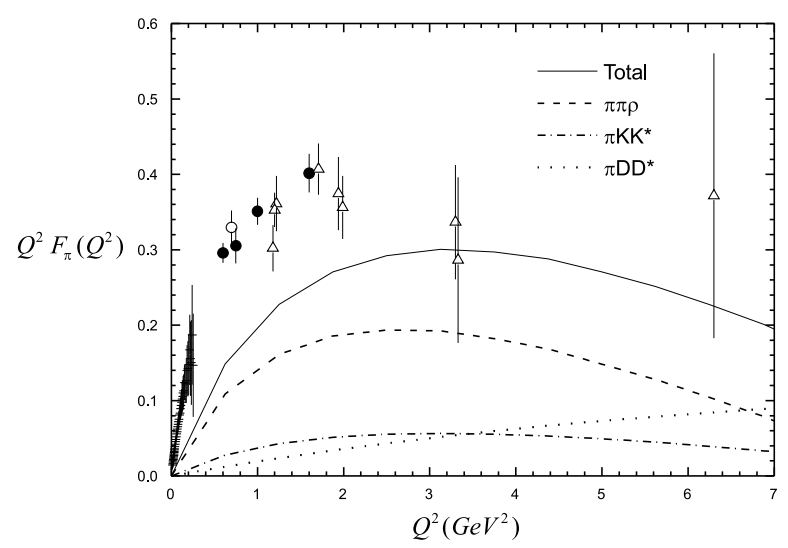

Figure 4. The same as Fig. 3, with $\Lambda_{\pi \pi \rho}=\Lambda_{\pi K K^{*}}=0.8 \mathrm{GeV}$ and $\Lambda_{\pi D D^{*}}=2.1 \mathrm{GeV}$.

As expected, we observe that the contribution from the $\mid \pi \rho>$ state is much larger than the others, due to the small masses involved. Surprisingly the $\mid D D^{*}>$ state gives a large contribution, which becomes more important at large $Q^{2}$. This is essentially due to the large value of the $D D^{*} \pi$ coupling. A possible interpretation of this fact is that at higher momenta, the photon can probe havier states.

Finally, we can compare the strength of the meson cloud in the pion (Figs. 3 and 4) and in the nucleon (Fig.1). We conclude that they have comparable magnitude, but in the case of the pion, the cloud is more important at intermediate momenta, while for the nucleon, the cloud becomes dominant at low momenta.

\section{References}

[1] J.D. Sullivan, Phys. Rev. D5, 1732 (1972).

[2] F. Carvalho, F. O. Durães, F. S. Navarra and M. Nielsen, Phys. Rev. D60, 094015 (1999).

[3] F. Carvalho, F. O. Durães, F. S. Navarra, M. Nielsen, and F.M. Steffens, Eur. Phys. J. C18, 127 (2000).

[4] W. Melnitchouk, J. Speth and A. W. Thomas, Phys. Rev. D59, 014033 (1999); A. Szczurek, H. Holtmann and J. Speth, 
Nucl. Phys. A605, 496 (1996); Nucl. Phys. A728, 182 (2003); K. Ohta, Phys. Rev. D35, 785 (1987); L. L. Barz et al., Nucl. Phys. A640, 259 (1998); Braz. J. Phys. 27, 358 (1997); H. Forkel et al., Nucl. Phys. A680, 179 (2000).

[5] G. A. Miller, nucl-th/0206027.

[6] F. Carvalho, F. O. Durães, F. S. Navarra and M. Nielsen, Phys. Rev. Lett. 86, 5434 (2001).

[7] see, for exeample, S. L. Zhu, Eur. Phys. J. AA, 277 (1999) and references therein.

[8] S. Ahmed et. al., Phys. Rev. Lett. 87, 251801 (2001).

[9] J. Volmer et. al. Phys. Rev. Lett. 861713 (2001)

[10] S. R. Amendolia et al., Nucl. Phys. B277, 168 (1986).

[11] P. Brauel et al., Z. Phys. C3, 101 (1979).

[12] C. J. Bebeck et al., Phys. Rev. D17, 1693 (1978). 